\title{
Optimization of Isolation and Identification Conditions of Glibenclamide Annotation
}

\author{
Ordabayeva Saule Kutymovna ${ }^{1}$, Karakulova Aizhan Shirinbekovna ${ }^{1}$, Serikbayeva Aigul Djumadullayevna ${ }^{1}$ and \\ Mirsoatova Mokhinur ${ }^{2}$ \\ 1. Department of Pharmaceutical and Toxicological Chemistry, South Kazakhstan State Pharmaceutical Academy, Shymkent 160019, \\ Kazakhstan
}

2. Science Club of Department of Pharmaceutical and Toxicological Chemistry, South Kazakhstan State Pharmaceutical Academy, Shymkent 160019, Kazakhstan

\begin{abstract}
A method for dispersive liquid-liquid microextraction of glibenclamide on model mixtures with urine has been developed. The extraction conditions have been optimized and the influence of extractants and dispersing agent for allocation of toxicant from biosubstrate has been experimentally established. The method of TLC (thin layer chromatography) screening in order to remove endogenous and exogenous substances has been developed. The method of IR-spectroscopy for confirmatory analysis has been used. The combination of the two methods of analysis allows identifying glibenclamide quickly and reliably isolated from bioliquid and reducing the risk of false-positive results.
\end{abstract}

Key words: Glibenclamide, dispersive liquid-liquid extraction, biological liquid, thin layer chromatography and infrared spectroscopy.

\section{Introduction}

In the arsenal of oral hypoglycemic drugs glibenclamide maintains its leading position as the most effective means because of its hypoglycemic activity. The effectiveness of the drug is due to its unique chemical structure, containing not only sulfonylureas ring, but also a benzamide moiety in the side chain. However, this feature may increase the risk of hypoglycemic conditions and have a negative effect on the cardiovascular system $[1,2]$.

Acute overdose of glibenclamide, as well as taking slightly higher doses over time, can lead to severe and prolonged life-threatening hypoglycemia [3-7].

The results of chemical-toxicological analysis of biological material or bioliquids have become important in the diagnosis of poisoning.

Preparation of the biological fluid for analysis

Corresponding author: Serikbayeva Aigul
Djumadullayevna, Ass. Prof., research fields: development of the method of analysis of drug isolation, identification and quantitative determination from the biological objects. generally involves the concentration step, extraction with a suitable solvent, chromatographic separation or sorption on the solid sorbent.

Reproducible and reliable results ensure proper sample preparation. In this connection, the issue of isolation test compounds from a biological matrix for the subsequent identification of their physicochemical methods is important.

In many studies, urine is selected as a biological object, because most of the exotoxines and their metabolites are excreted in the urine; furthermore, low contents of protein components therein and facilitates the isolation of compounds of interest further analysis.

The most common method to isolate analytes from urine is a LLE (liquid-liquid extraction). Nevertheless, the influence of endogenous substances in extracts of urine obtained using LLE significantly difficult to determine the test compounds by TLC (thin layer chromatography). An alternative is the DLLME (dispersive liquid-liquid microextraction) [8]. 
The aim of the study is to optimize the conditions of sample preparation and identification of glibenclamide expert analysis methods.

\section{Materials and Methods}

Standard sample substance of glibenclamide (India, LSR-series 0088753/09), solvents and chemicals, graded reagents were used. For identification we used infrared Fourier spectrometer InfraLUM FT-08 (Russia), the instrument kit for thin layer chromatography: chromatographic plate "Sorbfil PTLC-UV" (JSC "Sorbfil", Russia); UV chromatoscope, capillaries of $2 \mu \mathrm{L}, 5 \mu \mathrm{L}$ and $10 \mu \mathrm{L}$ (Russia).

\subsection{Isolation of Glibenclamide from Biological Fluid}

\subsubsection{Methods of Isolation}

Four $\mathrm{mL}$ of acetonitrile and $0.2 \mathrm{~mL}$ of chloroform was added to $5 \mathrm{~mL}$ of sample, mixed and centrifuged for $30 \mathrm{~min}$ at $3,500 \mathrm{rpm}$. We separated the lower portion and the resulting mixture was evaporated to dryness on a water bath. The dry residue was dissolved in $50 \mu \mathrm{L}$ of methanol, and chromatography was carried by upward manner.

\subsubsection{Preparation of Plates for Chromatography}

When using the acquired chromatography plates, you must first activate them. This is due to the fact that storage plates adsorbents adsorb not only the moisture, but also other substances contained in the air. When using untrained front plates "dirt", there is in the process of chromatography, which can interfere with the determination of substances having an $R_{f}$ value of large, but certain substances, such as water, can change the composition of the mobile phase, thereby changing the values obtained $R_{f}$. For preconditioning plate treated with $25 \%$ ammonia solution to the entire height of the plate, followed by drying the plate in an oven at a temperature $110 \sim 120{ }^{\circ} \mathrm{C}$ for $0.5 \sim 1 \mathrm{~h}$.

2.1.3 Method of Determination by Thin Layer Chromatography
On the start line in $15 \times 15$ sized chromatographic plates coated with MECS $10 \mu \mathrm{L}$ of a solution of glibenclamide and extraction of biological fluid. The plate with deposited samples were dried in air for 10 min, placed in a chamber with a mobile phase composition: chloroform-ethyl acetate-glacial acetic acid (4:6:0.1 by volume) and chromatographed by ascending manner. When the solvent front reaches the $12 \mathrm{~cm}$ plate was removed from the chamber, dried in air until complete disappearance of the odor solvents and viewed under UV light. Absorption zone after separation from the TLC plates were cut. Sorbent with these zones transferred to centrifuge tubes, $1 \mathrm{~mL}$ of acetone and centrifuged. Extraction with sorbent components performed three times using $1 \mathrm{~mL}$ of acetone. The extracts were combined and evaporated and the residue was used for further analysis by the spectroscopic method.

2.1.4 Preparation of Standard Sample Material Witness Solution of Glibenclamide

$0.15 \mathrm{~g}$ (accurately weighed) of glibenclamide was dissolved in $25 \mathrm{~mL}$ of mixture consisting of methylene chloride and methanol (1:1). Shelf-life of solution is one month.

2.1.5 Checking the Suitability of the Chromatographic System

The chromatographic system is considered suitable if a solution in the chromatogram standard sample material witness solution of glibenclamide is clearly visible spot, $R_{f}$ value which is $0.56 \pm 0.03$ (using mobile phase composition: chloroform-ethyl acetate-glacial acetic acid (4:6:0.1 by volume).

2.1.6 Determination of Glibenclamide by IR Spectroscopy

The infrared spectrum of the study medication were recorded on a Fourier transform IR spectrometer InfraLUM FT-08 using the method of pressing the test and standard samples in a potassium bromide tablet. For the preparation of such tablets small amount standard substance $(5 \sim 10 \mathrm{mg})$ and dry the obtained extract residue was mixed with potassium bromide, 
triturated in an agate mortar to a homogeneous powder which was transferred to the mold. The resulting tablets were placed in IR spectrometer and record spectra.

\section{Results and Discussion}

The role of DLLME method for fast and selective sample preparation to date increases. The advantages of the method are simultaneous isolation processes, concentration and purification given the right conditions. This reduces the time required for sample preparation, decrease solvent consumption and increase the accuracy of subsequent analysis. Due to the specific interactions can be selectively concentrate and extract each of the identified compounds that separate them from co-extractive components.

The second stage of the chemical-toxicological research is the identification of toxins in extracts obtained from different objects. Analysis of substances bioliquids is very challenging whose solution most appropriate to use HPLC-MS and GC-MS. But in practice, this approach meets a number of significant obstacles. For example, equipment for the implementation of data analysis is very expensive and there are not all the laboratories, so necessary to develop cheaper and more affordable medicines analysis methods of biological objects, which can be implemented on the equipment that is accessible to most analytical laboratories.

We have proposed an algorithm of identification of glibenclamide by using TLC and FT-IR spectrometry.

The analysis comprises the following steps:

(1) removing the analyte from the biological object;

(2) selection of the optimal conditions for TLC separation of the components;

(3) removing components zones TLC plates and identification libraries their IR spectra.

When selecting the optimal conditions, microextraction concentration determining its efficiency, as the test object urine used in an amount of $5 \mathrm{~mL}$ containing an analyte in an amount of $0.006 \mathrm{~g} / \mathrm{mL}$, which corresponds to a therapeutic dose of the drug.

Chloroform was used as an extraction agent because of its higher density than water, high extraction capacity in relation to the analytes, and its ability to form a stable two-phase system in the aqueous medium. These properties were determined in stability and some degree of concentration efficiency.

\subsection{The Selection of Extragent Volume}

The volume of chloroform administered ranged from $200 \mu \mathrm{L}$ to $500 \mu \mathrm{L}$. Microextraction performed at a constant amount of the dispersant $(2 \mathrm{~mL}$ of acetonitrile). Based on the chromatogram, glibenclamide found that the optimum extractant volume of $200 \mu \mathrm{L}$. In the chromatogram shows that by using the extractant in an amount of $200 \mu \mathrm{L}$ of analyte selectively split at $300 \mu \mathrm{L}$ clear additional spot on the chromatogram, not appropriate glibenclamide, and the use of an extractant in an amount of $400 \mu \mathrm{L}$ and $500 \mu \mathrm{L}$, one of ballast substances drawn on the front line and reduce the sharpness of separation spots test substance. From this, it can be concluded that increasing the volume of extractant leads to deterioration of the separation efficiency of the analyte (Fig. 1).

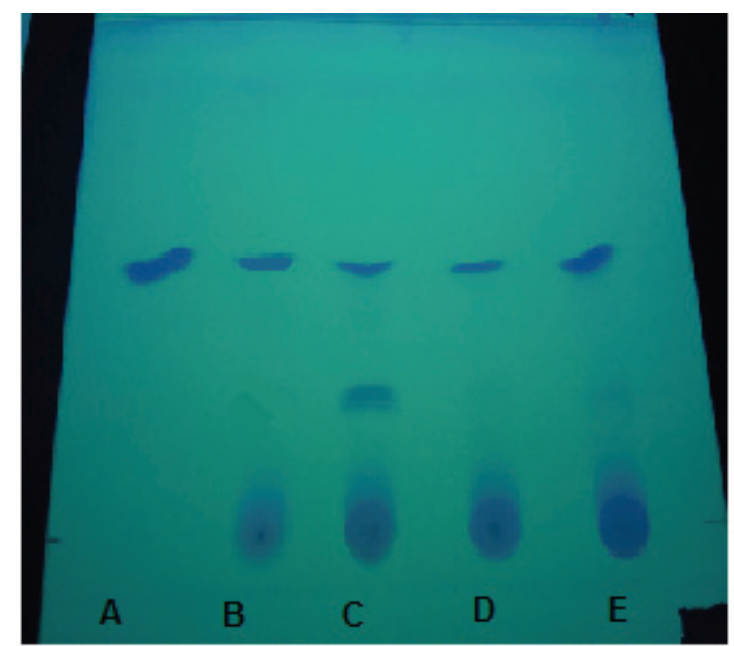

Fig. 1 The zones of absorption of glibenclamide using chloroform in an amount of $200 \sim 500 \mu \mathrm{L}$.

A - standard of glibenclamide; B, C, D, E-test substances on the rising volume of extracting agent $(200 \mu \mathrm{L}, 300 \mu \mathrm{L}, 400 \mu \mathrm{L}$ and $500 \mu \mathrm{L})$. 


\subsection{The Volume of Dispersant}

The acetonitrile is used as a dispersant. The volume of the dispersant was varied from $1 \mathrm{~mL}$ to $4 \mathrm{~mL}$. It is found that when the amount of the dispersant in an amount of $4 \mathrm{~mL}$ of spots in the chromatogram obtained without the presence of background substances. With a volume of $1.0 \mathrm{~mL}, 2.0 \mathrm{~mL}$ and $3.0 \mathrm{~mL}$ of the hood in addition to the test, compounds contains a significant amount co-extractive substances, which makes it difficult to identify the analyte (Fig. 2).

TLC was used for further purification and identification of glibenclamide. In order to select the chromatographic conditions to enable to achieve selective separation of glibenclamide, studied its chromatographic behavior in different solvent systems.

Selection of the mobile phase composition should be adjusted to the eluent strength, selectivity and chemical stability of its components. It influences the mobile phase of different composition and polarity on the mobility of glibenclamide and selectivity of the chromatographic system.

Glibenclamide mobility was evaluated based on the quantities $R_{f}$, which are in the $0.2 \sim 0.8$ range, which is optimal.

Chromatography was carried out in different solvent systems: chloroform-acetone (9:1), 96\% ethanol-butanol-25\% ammonia solution (35:10:5), $96 \%$ ethanol-water-25\% ammonia solution (24:4:2.5), toluene-ethyl acetate- $96 \%$ ethanol (8:0.5:1), chloroform-ethyl acetate-glacial acetic acid (4:6:0.1), benzene-ethyl acetate-acetic acid-acetone (65:6:12:30) hexane-chloroform- $96 \%$ ethanol-acetic acid (8:8:1:1) to "Sorbfil" plates with a fixed sorbent bed.

The mobile phases of benzene-ethyl acetate-acetic acid-acetone (65:6:12:30), and chloroform-ethyl acetate-glacial acetic acid (4:6:0.1) was not possible to obtain a precise contour chromatograms with stains which drastically reduces the sensitivity determination. Moreover, a clear separation of spots in these systems also tends not to be observed.

The solvent systems of $95 \%$ ethanol-water- $25 \%$ ammonia solution (24:4:2.4 by volume) and chloroform-acetone (9:1) glibenclamide is not sufficiently chromatographic mobility, remaining mostly near the starting line.
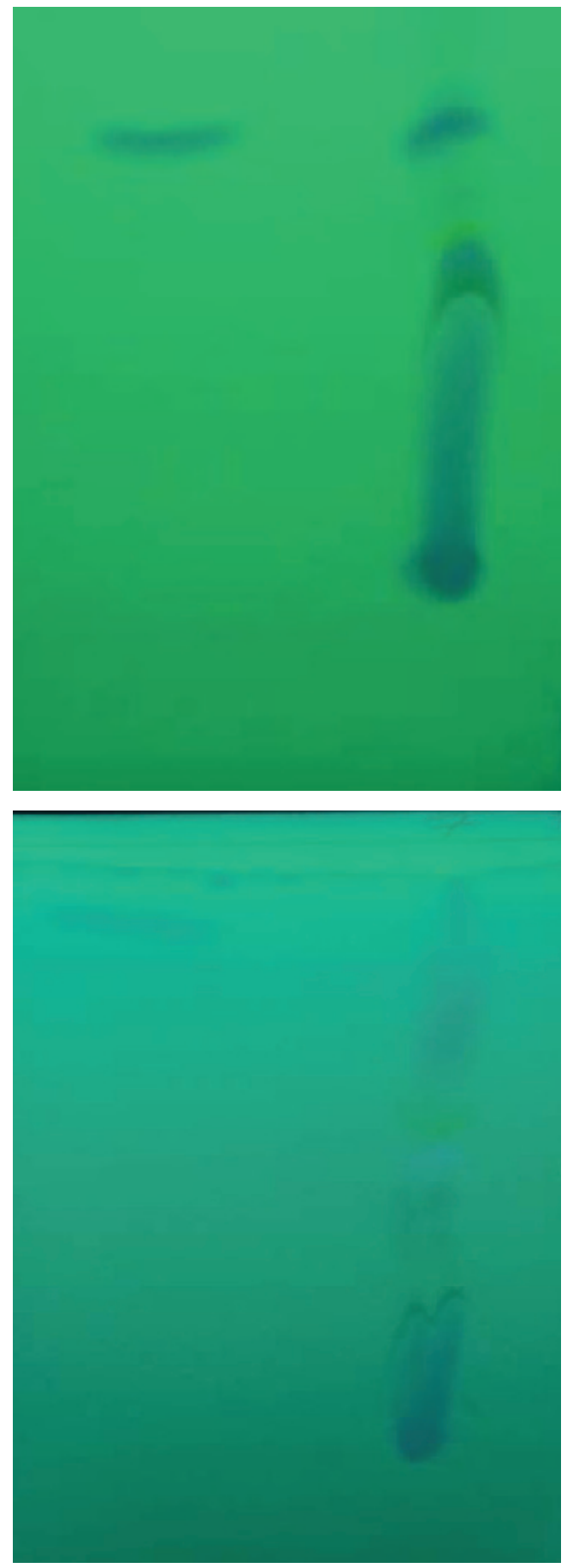

Fig. 2 The zones of absorption of glibenclamide spots when using $2 \mathrm{~mL}$ and $3 \mathrm{~mL}$ of dispergator. 
On the basis of experimental data, we have selected the system consisting of chloroform-ethyl acetate-glacial acetic acid (4:6:0.1), which are compact glibenclamide spots have clear boundaries and a sufficient degree of separation $\left(R_{f}, 0.56\right)$.

Next, this mobile phase was used to identify glibenclamide isolated after DLLME method developed by us. Optimized conditions allowed the procedure to reduce the intensity of the distribution of ballast substances in the chromatogram. In the chromatogram obtained clearly show that the intensity of the background in the chromatogram is low, and the spot of the analyte divided compact and coincides with the location of the areas all glibenclamide (Fig. 3).

To evaluate the results, an absorption zone with a spot test substance removed together with the stationary phase dissolved in acetone and filtered through a glass filter and evaporated at $40 \sim 50{ }^{\circ} \mathrm{C}$. The resulting residue was triturated with dry potassium bromide, and the spectrum was recorded. IR spectra showed that the selected method needs to be adjusted, as not all areas are equally accessible wavelengths due to the intrinsic absorption of silica gel.

In order to eliminate extraneous effects on sorbent glibenclamide identification results by IR spectroscopy, the above described procedure was modified: the substances after separation on the chromatographic plate the absorption zone was excised from the TLC plates, transferred to centrifuge tubes, $1 \mathrm{~mL}$ of acetone, centrifuged. The extraction of the analyte from the sorbent was performed three times using $1 \mathrm{~mL}$ of acetone. The extracts were combined, evaporated and the residue was triturated with potassium bromide. The resulting mixture was compressed into a tablet, and the infrared spectrum recorded. Simultaneously compressed tablet with potassium bromide and witness standard substance.

IR glibenclamide isolated from urine and extracted with acetone TLC plate at the conditions described above, it revealed the presence of several characteristic bands corresponding to specific types of oscillations of different structural fragments standard substance witness molecule (Table 1).

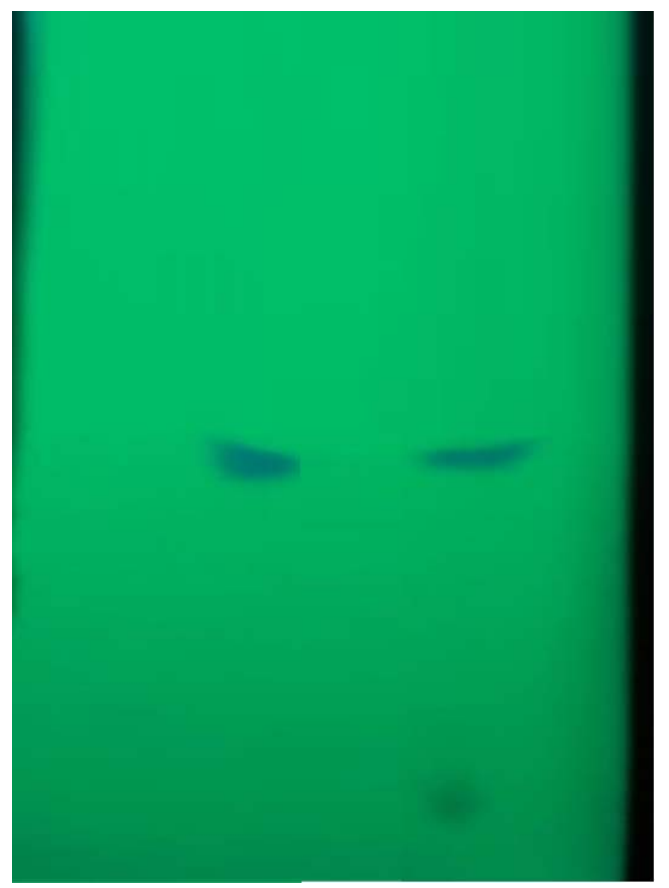

Fig. 3 The zones of adsorption of glibenclamide spots when using $4 \mathrm{~mL}$ of dispergator.

Table 1 Characteristics of IR spectra of a standard substance of glibenclamide and glibenclamide substance isolated from urine.

\begin{tabular}{lll}
\hline \multirow{2}{*}{ Types of ranges } & \multicolumn{2}{c}{ Maximums of characteristic stripes $\left(\mathrm{cm}^{-1}\right)$} \\
\cline { 2 - 3 } & Glibenclamide, extracted from urine & Standard solution of glibenclamide \\
\hline $\mathrm{C}=\mathrm{O}$ valent ranges & 1,618 & 1,618 \\
$-\mathrm{CH}_{2}$ - deformational ranges & $1,479,1,461,1,449,1,436$ & $1,479,1,461,1,450,1,437$ \\
$-\mathrm{CONH}-\mathrm{R}$ valent ranges & $3,371,3,319,1,716$ & $3,371,3,319,1,718$ \\
$-\mathrm{SO}_{2}$ - valent ranges & $1,342,1,307,1,160$ & $1,342,1,308,1,160$ \\
$-\mathrm{C}-\mathrm{N}-$ valent ranges & $1,244,1,182$ & $1,244,1,182$ \\
\hline
\end{tabular}




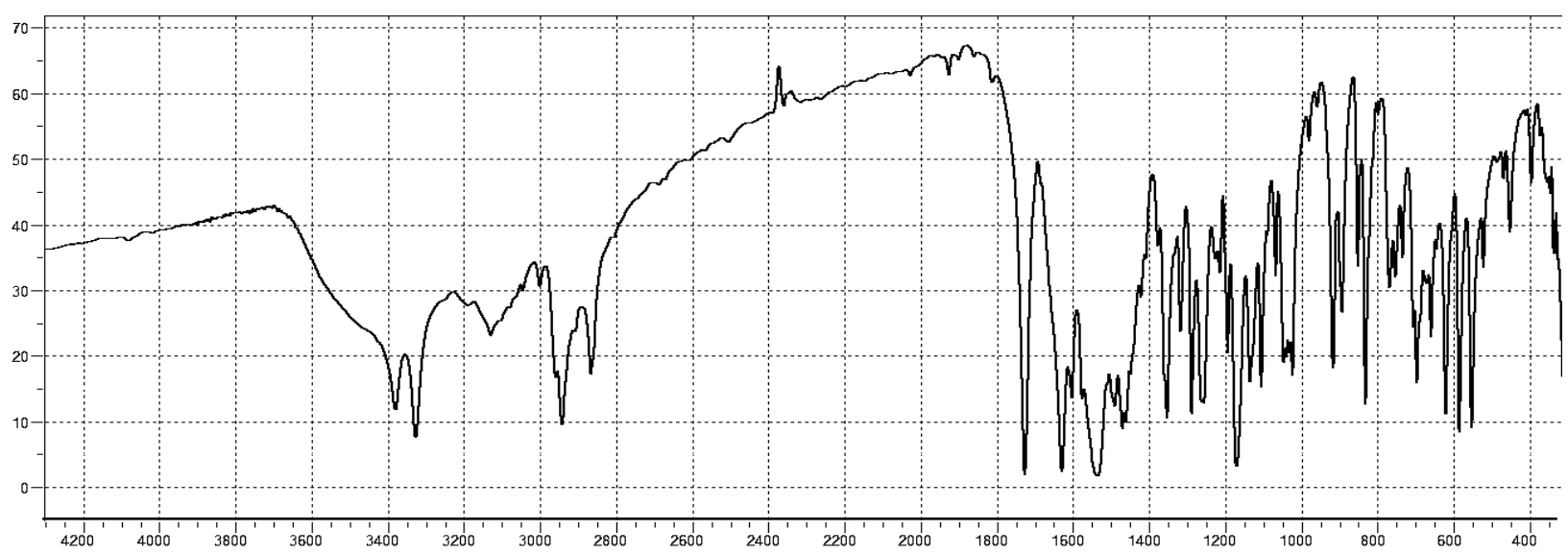

(a)

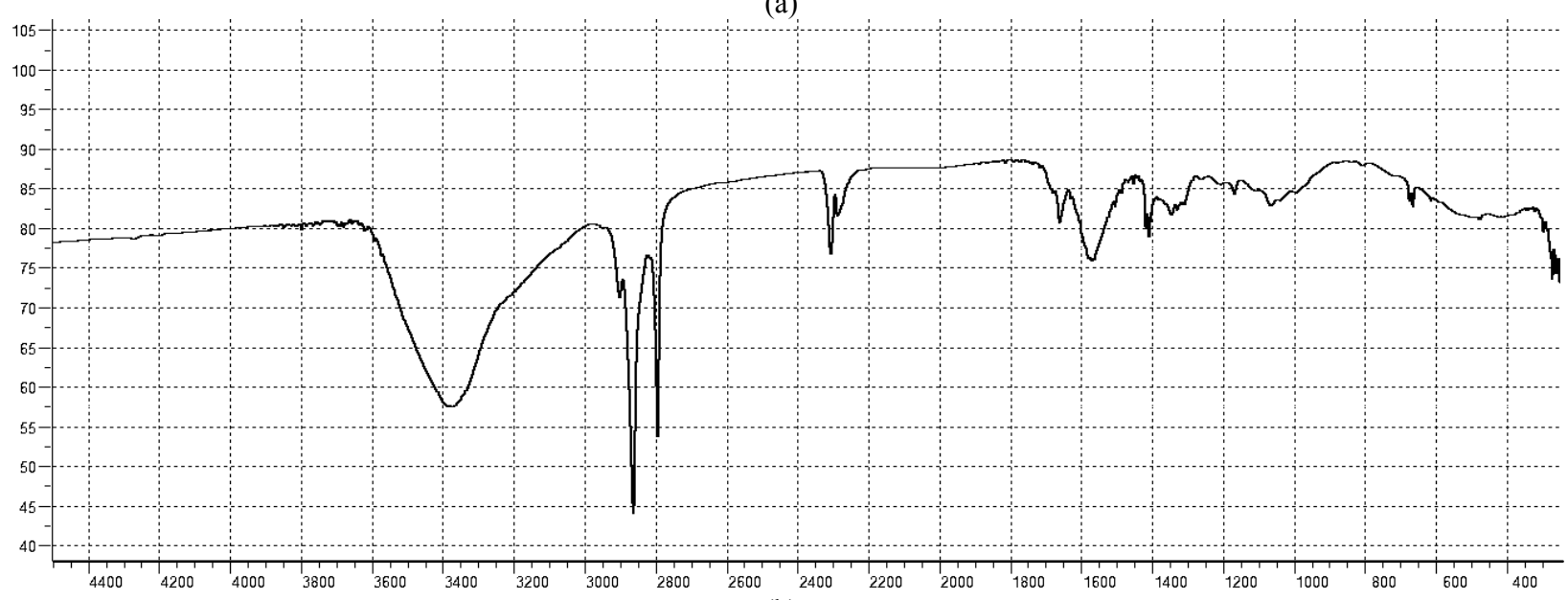

(b)

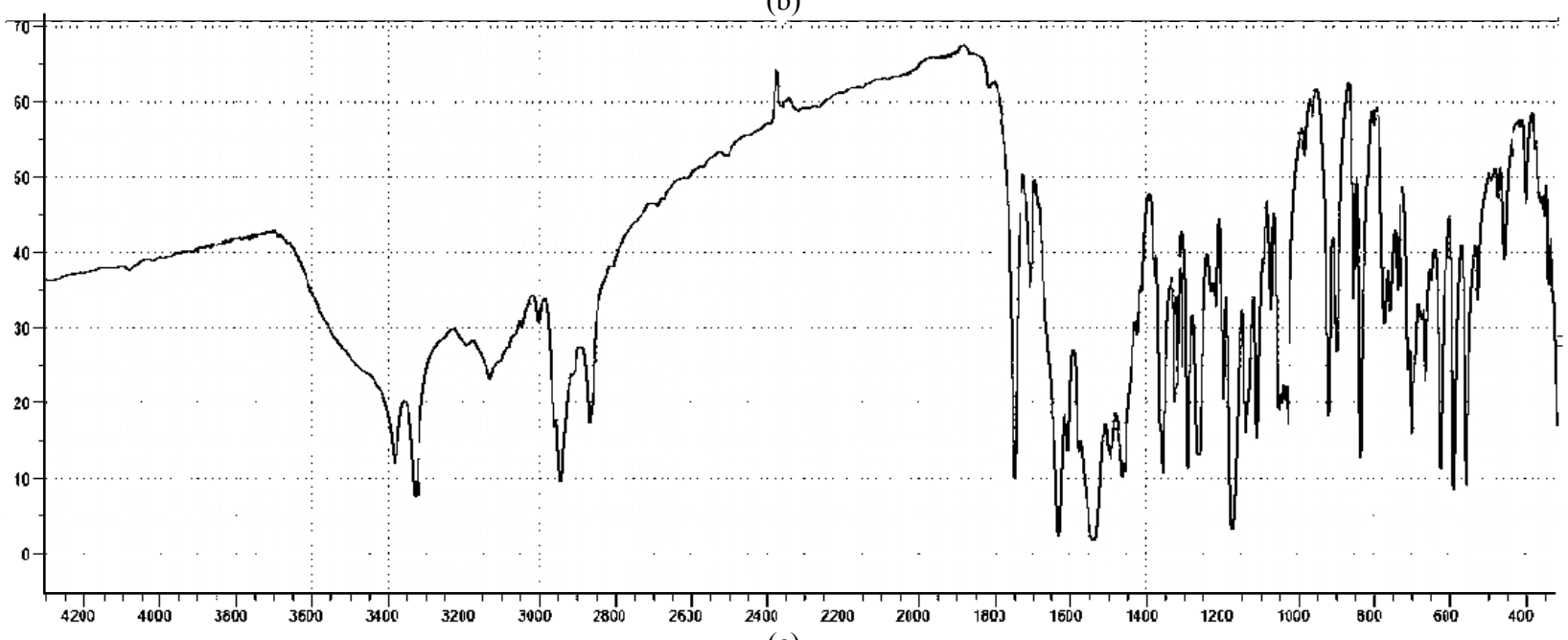

(c)

Fig. 4 IR spectra of glibenclamide (units in $\mathrm{cm}^{-1}$ ): (a) spectra of standard sample of glibeclamide; (b) spectra of glibeclamide with eluent; (c) spectra of glibeclamide after extracting with acetone.

\section{Conclusions}

Thus, as a result of our studies optimized conditions of extraction glibenclamide urine. When carrying out dispersion using the isolation liquid-liquid microextraction good contact is obtained purified of ballast material with a compact test compound division in selected experimental phase consisting of 
chloroform-ethyl acetate-glacial acetic acid (4:6:0.1 by volume). DLLME method simultaneously conducted to isolate the process of concentration and purification. This has reduced the time required for sample preparation, reduce the consumption of solvents, improve the accuracy of subsequent analysis. Identification of glibenclamide using a combination of TLC and FT-IR spectrometry. Optimized conditions for removal of the analyte to remove IR spectra and this causes principal possibility of using infrared spectroscopy to identify glibenclamide isolated from urine in therapeutic concentrations.

\section{References}

[1] Duckworth, W., Abraira, C., Moritz, T., Reda, D., Emanuele, N., Reaven, P. D., et al. 2009. "Glucose Control and Vascular Complications in Veterans with Type 2 Diabetes." N. Engl. J. Med. 360: 129-39.

[2] Clemens, K. K., McArthur, E., Dixon, S. N., Fleet, J. L., Hramiak, I., and Garg, A. X. 2015. "The Hypoglycemic Risk of Glyburide (Glibenclamide) Compared with Modified-Release Gliclazide." Can J. Diabetes 39 (4): 308-16.

[3] Gangji, A. S., Cukierman, T., Gerstein, H. C., Goldsmith,
C. H., and Clase, C. M. 2007. "A Systematic Review and Meta-analysis of Hypoglycemia and Cardiovascular Events: A Comparison of Glyburide with Other Secretagogues and with Insulin." Diabetes Care 30: 389-94.

[4] Kane, M. P., Abu-Baker, A., and Busch, R. S. 2005. "The Utility of Oral Diabetes Medications in Type 2 Diabetes of the Young." Curr. Diabetes Rev. 1 (1): 83-92.

[5] Mowry, J. B., Spyker, D. A., Cantilena Jr, L. R., McMillan, N., and Ford, M. 2013. Annual Report of the American Association of Poison Control Centers' National Poison Data System (NPDS): 31st Annual Report. Clin Toxicol (Phila).

[6] Mowry, J. B., Spyker, D. A., Cantilena Jr, L. R., Bailey, J. E., and Ford, M. 2012. Annual Report of the American Association of Poison Control Centers' National Poison Data System (NPDS): 30th Annual Report. Clin Toxicol (Phila).

[7] Bronstein, A. C., Spyker, D. A., Cantilena Jr, L. R., Rumack, B. H., and Dart, R. C. 2011. Annual Report of the American Association of Poison Control Centers' National Poison Data System (NPDS): 29th Annual Report. Clin Toxicol (Phila).

[8] Zgoła-Grześkowiak, A., and Grześkowiak, T. 2011 "Dispersive Liquid-Liquid Microextraction." Trends Anal. Chem. 30: 1382-99. 